E3S Web of Conferences 1, 37002 (2013)

DOI: $10.1051 / \mathrm{e} 3$ sconf/20130137002

(C) Owned by the authors, published by EDP Sciences, 2013

\title{
Preconcentration of Arsenic in Water Samples Using the Composition-Induced Phase Separation Method and Determination by ETAAS
}

\author{
M. Güçoğlu ${ }^{1}, M$. Efeçınar ${ }^{2}$ and N. Şatıroğlu ${ }^{3}$ \\ ${ }^{1}$ Department of Chemistry, Hacettepe University, Ankara, TURKEY, melekalp@hacettepe.edu.tr \\ ${ }^{2}$ Department of Chemistry, Hacettepe University, Ankara, TURKEY, melise@hacettepe.edu.tr \\ ${ }^{3}$ Department of Chemistry, Hacettepe University, Ankara, TURKEY, nurayo@hacettepe.edu.tr
}

\begin{abstract}
A new phase transition microextraction method was developed for determination of trace amount of arsenic ions in water samples in this work. The method is based on the critical point of miscibility of solvents. In this method the mixed solution of sample and organic solvent is initially homogeneous but is separated into two phases by adding a secondary solvent (modifier). In acidic medium As(V) was complexed with ammonium molybdate, this complex was quantitatively extracted to the mixture of organic solvent (acetonitrile/methyl isobutyl ketone) before ETAAS determination. Total inorganic arsenic (III, V) was extracted similarly after oxidation of $\mathrm{As}(\mathrm{III})$ to $\mathrm{As}(\mathrm{V})$ with nitric acid. Concentration of As(III) was calculated by difference in the concentration between total arsenic and $\mathrm{As}(\mathrm{V})$. Optimization of the experimental conditions and instrumental parameters was investigated in detail. A detection limit of $0.05 \mu \mathrm{gL}^{-1}$ with enrichment factor of 85 was achieved for only $5 \mathrm{~mL}$ of sample. The analytical curve was linear in the concentration range of $0.25-4.00 \mu \mathrm{gL}^{-1}$. Relative standard deviation (RSD) for 10 replicate determinations of $2.0 \mu \mathrm{gL}^{-1}$ of $\mathrm{As}(\mathrm{V})$ was $4,1 \%$. The method was successfully applied to preconcentration and determination of arsenic in real water samples.
\end{abstract}

Key words: Arsenic, preconcentration, composition-induced phase separation, ETAAS

\section{Introduction}

Arsenic is a ubiquitous element in the environment originating from natural sources as well as human activities, and has been identified as a public health problem because it has serious toxic effects even at low exposure levels (Mazumder, 1997). Different forms of arsenic have different toxicity and inorganic arsenic is more toxic than organic arsenic and As(III) is more toxic than As(V) (Majid et al., 2006). There are many reports in literature about the higher risks of skin, lung, liver and kidney cancer. The World Health Organization's (WHO) maximum permissible contaminant level of arsenic in drinking water is $10 \mu \mathrm{gL}^{-1}$. Therefore, the development of a sensitive and simple method for the determination of trace arsenic in water samples is significant.

The development of separation and preconcentration procedure prior to trace element determination has been explored in considerable depth in recent decades. Several procedures have been used for the separation and preconcentration of contaminants from environmental samples; liquid-liquid extraction, co-precipitation, solid phase extraction.

Liquid-liquid extraction is a unit operation for separating one or more components from a liquid mixture. In recent years, research efforts in separation science and related research fields have focused on miniaturization of sample preparation process.

Liquid-liquid microextraction is a modified solvent extraction method and in this method organic phase-aqueous phase ratio $(\mu \mathrm{L}: \mathrm{mL})$ is greatly reduced comparing with the other extraction methods. It needs low volume of the sample and has sufficient preconcentration factor.

Therefore, this method is very useful for the separation and preconcentration of metal ions after the formation of sparingly water-soluble complexes (Pena-Pereira et al., 2009).

A novel liquid extraction process, named phase transition extraction, which is based on using solvents that, together with the liquid to be extracted, form a solution with a critical point of miscibility was developed 
by Ullmann et al. (Ullmann et al., 1995). In this process, the phase transition of partially miscible mixtures is achieved by adding a modifier which is insoluble in native (water) solvent. The method carried out isothermally by changing the mixture composition instead of its temperature is called as composition-induced phase separation (CIPS) (Gupta et al., 1996) or solvent-induced phase transition extraction (SIPTE) (Liu et al., 2010). This extraction method has been applied for the separation of organic compounds from matrix in literature. In this work, first time CIPS methodology was successfully applied for the microextraction of arsenic in water samples as a prior step to their determination by ETAAS.

\section{Materials and Methods}

All chemicals used in this work were of analytical grade and all solutions were prepared with deionized water $(18.1 \mathrm{M} \Omega \mathrm{cm})$ obtained from a Barnstead, Nanopure Diamond purification system. As(V) and As(III) stock standard solutions $\left(1000 \mathrm{mgL}^{-1}\right)$ were prepared from $\mathrm{Na}_{2} \mathrm{HAsO}_{4} \cdot 7 \mathrm{H}_{2} \mathrm{O}$ (Sigma-Aldrich) and $\mathrm{As}_{2} \mathrm{O}_{3}$ (Fisher Scientific). Ammonium heptamolybdate tetrahydrate $\left(\left(\mathrm{NH}_{4}\right)_{6} \mathrm{Mo}_{7} \mathrm{O}_{24} \cdot 4 \mathrm{H}_{2} \mathrm{O}\right)$ (Merck) was used as a complexing agent. Ammonium molybdate was prepared in $\mathrm{H}_{2} \mathrm{SO}_{4}$. $\mathrm{H}_{2} \mathrm{SO}_{4}, \mathrm{HCl}$ and $\mathrm{HNO}_{3}$ were used for adjusting $\mathrm{pH}$ of samples. In addition concentrated nitric acid was used as an oxidant agent for converting $\mathrm{As}(\mathrm{III})$ to $\mathrm{As}(\mathrm{V})$. The organic extractant was mixture solution of acetonitrile and methyl isobutyl ketone. $\operatorname{Pd}\left(\mathrm{NO}_{3}\right)_{2}$ (Sigma-Aldrich) was used as a chemical modifier in ETAAS. Measurements were carried out on Perkin-Elmer AAnalyst 800 atomic absorption spectrophotometer equipped with THGA prolytic coated graphite furnace atomizer and arsenic electrodeless discharge lamp.

The test solution which is containing arsenic and complexing agent is first mixed with acetonitrile; then a drop of MIBK is added as the modifier to induce phase separation of the mixture. The separated organic phase with the analyte extracted can be directly injected to the ETAAS.

It is known that only $\mathrm{As}(\mathrm{V})$ forms complex with ammonium molybdate, but not As (III). In this method, after $\mathrm{As}(\mathrm{V})$ was complexed with ammonium molybdate, organic solvents were added into $5 \mathrm{~mL}$ of aqueous sample (final $\mathrm{pH}$ of this arsenic sample was 0.8-1.0) and added volume of organic solvents was in the $\mu \mathrm{L}$ level. The mixture was stirred using vortex for about 5 minutes. Separation of the aqueous and organic phases was accomplished approximately in 30 seconds. After extraction, the $60 \mu \mathrm{L}$ of organic phase enriched with arsenic was taken by a micropipette and determined by ETAAS. After simple oxidation with nitric acid, converting As(III) to As(V), total arsenic was extracted and determined similarly (Sounderajan et al., 2007). The concentration of As(III) was calculated by difference in the concentration between total arsenic and $\mathrm{As}(\mathrm{V})$.

\section{Results and Discussion}

In this method acetonitrile and MIBK were used as organic solvents. Acetonitrile is completely miscible with water but MIBK and water form a broad miscibility gap. Therefore MIBK is used as a secondary solvent or modifier.

Several factors that influence the microextraction efficiency and ETAAS signal, such as $\mathrm{pH}$, concentration of chelating agent, extraction time, organic solvents and extraction solvents, organic phase ratio, pyrolysis and atomization temperature were investigated.

Important parameters such as the linear range, calibration graph, precision, detection limit, recoveries, and enrichment factor were determined to evaluate the method performance. The calibration graph was linear in the range of $0.25-4.0 \mu \mathrm{gL}^{-1}$ with correlation coefficient of 0.9983 under the optimum conditions of the recommended procedure. The detection limit was 0.05 $\mu \mathrm{gL}^{-1}$ and the relative standard deviation $(\mathrm{n}=10)$ at 2 $\mu \mathrm{gL}^{-1} \mathrm{As}(\mathrm{V})$ concentration was $4.1 \%$. The enhancement factor defined as the slope ratio of two calibration curves with microextraction and without microextraction was 85 .

In order to verify the accuracy of the proposed procedure, the method was applied to the determination of $1.36 \mu \mathrm{gL}^{-1}$ As in standard reference material, SLEW-3 Estuarine water. The presented procedure was applied to the speciation of arsenic in a tap water, sea water, drinking water and ground water. Various amounts of $\mathrm{As}(\mathrm{V})$ and $\mathrm{As}(\mathrm{III})$ were also spiked to these samples. A good agreement was obtained between the added and measured analyte amounts. The conventional LLE and CIPS methods were also applied to the standard solutions and obtained results were compared.

\section{Conclusion}

We have presented a fast, reliable, sensitive and accurate method, which benefits from a very low detection limit, for the determination of $\mathrm{As}(\mathrm{V})$ and total arsenic in aqueous solutions. Using the proposed method, arsenic can be determined with a very high sensitivity and relatively good reproducibility in real water samples such as tap water, sea water, ground water and drinking water.

\section{References}

Gupta R, Mauri R, Shinnar R. Liquid-Liquid Extraction Using the Composition-Induced Phase Separation Process, Ind. Eng. Chem. Res., 1996, 35, 236.

Liu G, Zhou N, Zhang M, Li S , Tian Q, Chen J, Chen B, $\mathrm{Wu}$ Y, Yao S. Hydrophobic solvent induced phase transition extraction to extract drugs from plasma for high performance liquid chromatography-mass spectrometric analysis, Journal of Chromatography A, 2010, 1217, 243.

Majid E, Harapoviç S, Liu Y, Male K.B, Luong J.H.T. Electrochemical determination of arsenite using a 
gold nanoparticle modified glassy carbon electrode and flow analysis, Anal. Chem., 2006, 78 , 762.

Mazumder N.G. Chronic arsenic toxicity in West Bengal, Curr. Sci., 1997, 72, 114.

Pena-Pereira F, Lavilla I, Bendicho C. Miniaturized preconcentration methods based on liquid-liquid extraction and their application in inorganic ultratrace analysis and speciation: A review, Spectrochimica Acta Part B, 2009, 64, 15.

Shemirani F, Baghdadi M, Ramezani M. Preconcentration and determination of ultra trace amounts of arsenic (III) and arsenic (V) in tap water and total arsenic in biological samples by cloud point extraction and electrothermal atomic absorption spectrometry, Talanta, 2005, 65, 887.

Sounderajan S, Udas A.C., Venkataramani B, Characterization of arsenic (V) and arsenic (III) in water samples using ammonium molybdate and estimation by graphite furnace atomic absorption spectroscopy, Journal of Hazardous Materials, 2007, $149,238$.

Ullmann A, Ludmer Z, Shinnar R. Phase transition extraction using solvent mixtures with a critical point of miscibility, AIChE J., 1995, 41, 488. 